\title{
Context-aware Semantic Infrastructure for IPv6-based Smart Home
}

\author{
Zhenyu Liu \\ National Engineering Laboratory for Digital Home Network \\ Qingdao, China \\ Hui Yang \\ College of Information Science and Engineering \\ Ocean University of China, \\ Qingdao, China \\ yy_ouc@163.com
}

\author{
Gui Chen \\ State Key Laboratory of Digital Household Appliances \\ Qingdao, China \\ Yang Pan \\ State Key Laboratory of Digital Household Appliances \\ Qingdao, China
}

\begin{abstract}
As the urgent need for unprecedented level of convenience, enjoyment and quality of lives becomes ever more apparent, interest in smart homes is on the rise. Recent advances in computer and communication technologies have offered many promising solutions to the realm of assistive living. However, to allow a smart home promotes users' comfort and entertainment through the management of technology within the home and connections to the world beyond, applications in such environment must be context aware so that they can anticipates and responds to the need of the users as they move about in their environments. Nevertheless, there is a major gap between practice and aspiration. In this paper, we propose context-aware semantic infrastructure - a novel concept whose aim is to move from the current state-of-the-art of smart home technologies to the future infrastructure that is needed to support the full richness of the smart home vision. We focus predominantly on the methodology of OWL-based semantic modeling, content generation and management. The framework exploits semantic web and context-awareness technologies to support context representation and storage, strategy querying, semantic reasoning of contexts and knowledge management in smart homes. This context-aware semantic infrastructure can provide a guide for the subsequent research.
\end{abstract}

Keywords-semantic web; context aware; smart home; OWL; case-based reasoning

\section{INTRODUCTION}

The core concept of pervasive computing [1] is that computing can occur everywhere and anywhere, despite much of the time it is invisible to users. Smart Home is a specific implementation of pervasive computing, where physical and computing entities are incorporated together to become a rule-based reasoning smart space, providing essential environment information and technical support for various entities (users or appliances). A number of projects and research have developed ubiquitous home network models, such as „Aware Home ${ }^{e e}$, developed by the Georgia Institute of Technology based on ubiquitous computing that senses and recognizes potential crises, to assist declining elderly memory and find behavioral trends and Place Lab, a part of House project of MIT which monitors the activity and vital signs of the residents, controls energy expenditure, and provides entertainment, learning, and communication using ubiquitous sensors and wearable systems[2].

Recently, with the emergence of IPv6, everything from appliances to automobiles can be interconnected more conveniently than ever before. Smart home is therefore to become a reality. First, IPv6 has a larger address space, which can offer every appliance or digital device a unique IPv6 address. Second, IPv6 hosts can configure themselves automatically when connected to an IPv6 network using address auto-configuration. Third, Internet Protocol Security (IPsec), which provides confidentiality, authentication and data integrity, is baked into IPv6. Fourth, IPv6 supports multicast rather than broadcast. Multicast allows bandwidth-intensive packet flows (like multimedia streams) to be sent to multiple destinations simultaneously, saving network bandwidth [3].

However, applications in such environments must be context aware so that they can adapt to rapidly changing conditions as users move about in their environments [4] [5].

To address this issue, we developed a context-aware infrastructure inspired by the Semantic Web which helps computers and people work better together by giving content well-defined meanings, as is described in Part II. In Part III, we introduce a knowledge base management platform as a unified semantic interface for smart space application resources and Part IV makes a corresponding summary.

\section{SEMANTIC WEB INFRASTRUCTURE}

\section{A. Categories of context in smart home}

The dynamic nature of smart spaces poses the challenge that intelligent entities must be context aware so that they can adapt to rapidly changing conditions. Context awareness refers to the idea that computers can both sense, and react based on their environment. Devices may have information about the circumstances under which they are able to operate and based on rules, or an intelligent stimulus, react accordingly. Context can describe static and dynamic 
information of relevant entities during the interaction process among environments, users and applications. In terms of smart space homes, context can be divided into three categories [6]:

1) Computing context, which is composed of computing capability, storage capability, bandwidth, error rate, connection establishment time, cost, safety requirement, competition, delay etc., may lead to changes of protocols, executable program behaviors and functionalities.

2) Environment context, which consists of social conditions, physical conditions etc., e.g. temperature, brightness, humidity.

3) User context, which includes labeling of the individual, location, behavior and preferences etc.

\section{B. Semantic web infrastructure}

Semantic web is capable of organizing various resources reasonably and effectively, and implementing computer automatic processing through rule-based reasoning. Heterogeneous resources can be formally and explicitly described based on ontology in semantic web.

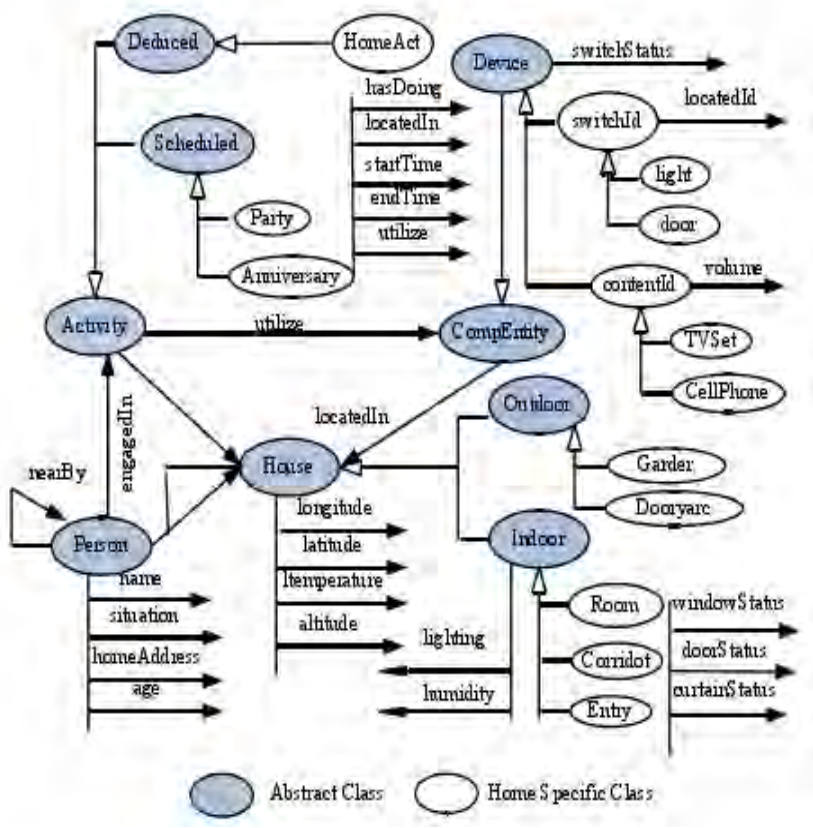

Figure 1. Smart home context model

Therefore, ubiquitous computing entities can share a common understanding of context structure, which makes interoperability possible. Since the semantic web can acquire a wide range of contextual information, it is feasible to develop various specific management tools and allow user to pre-define their ontology set and determine the relevant contextual information[7]. Hence, it is necessary for us to research on the open semantic web infrastructure and software platform.

The semantic web infrastructure we proposed is mainly made up of knowledge base and semantic rule inference engine, as shown in Fig .2.

1) Knowledge base, which is based of four types of knowledge[8][9]: a) Static knowledge, which is irrelevant with context and describes basic concepts and attributes of the entities in the smart space system.

b) Dynamic knowledge, which is relevant with context and describes the changing resources and device control strategies, e.g. shut down the illumination system when I leave home.

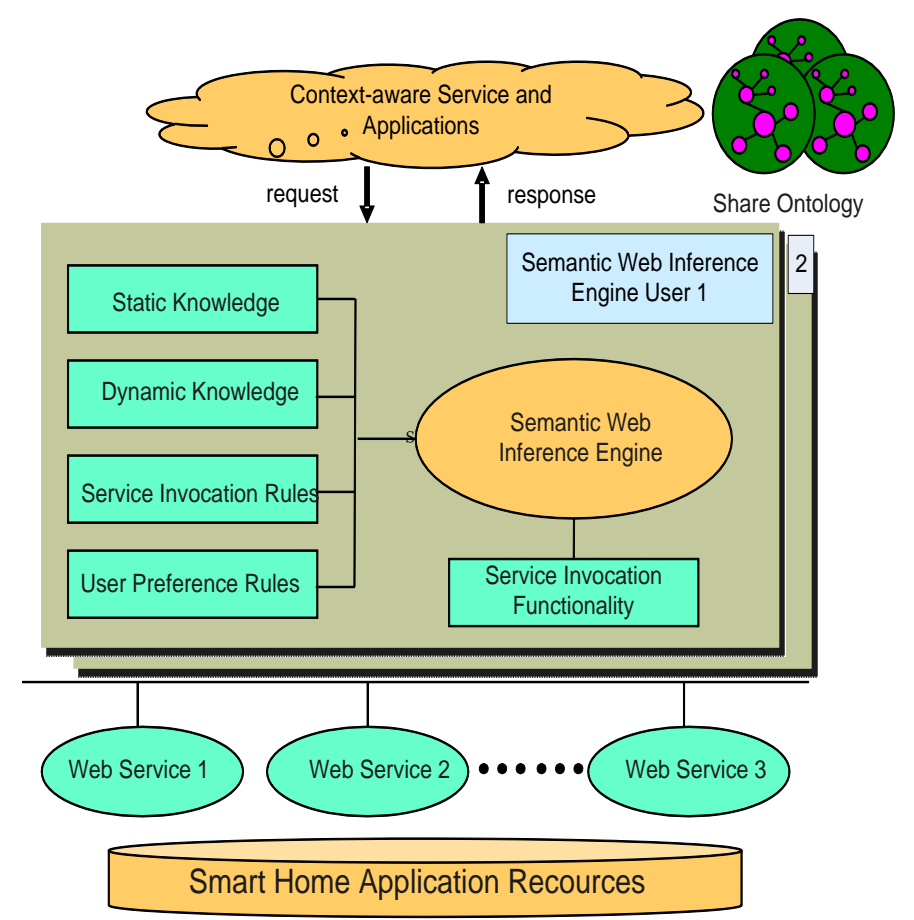

Figure 2. Semantic web infrastructure

c) Service invocation rules, which build mappings between context attributes and usable application resources in smart home, and facilitate other entities in the system to visit these resources with inference engine e's support. The mapping is not necessary to be a restricted one-to-one mapping, which means context attribute can select and invoke one of the services to implement the same functionality.

d) User preferences rules, e.g. when user turns on the television, it is automatic to switch to the channel as the user preference defined.

2) Reference engine.The reference engine can apply automatic control mechanism according to these four types of knowledge. We designed the semantic web infrastructure as follows [10].

a) Transferred the knowledge into the form of first-order predicate logic.

b) Constructed ontology with $O W L-D L$; 
c) Build semantic web knowledge base using RDF/RDFS language.

\section{d) Use CLIPS as semantic Web inference engine.}

\section{SEMANTIC WEB KNOWLEDGE BASED}

\section{MANAGEMENT PLATFORM}

Fig .4 shows the knowledge base management platform we designed with learning, conversation and interpretation functionalities, which provides a unified semantic interface for applicable resources in smart home. On the platform, all

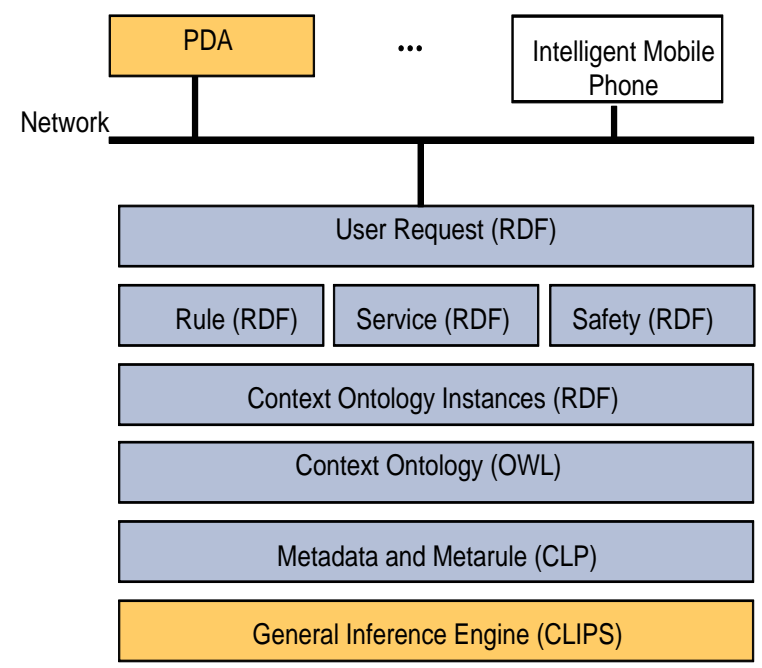

Figure 3. Implementation of the semantic web infrastructure

kinds of smart space related contextual information resources are described as a single semantic Web service. The platform is responsible for the management and maintenance of the contextual resources Web service catalogue. This platform provides a man-machine interface and helps family users to create, convert and store knowledge and strategies. Due to the information and knowledge asymmetry between system and users, the implementation mechanism of the management platform should include strategy-based functionalities of conversation, learning and interpretation to lower the difficulties of understandings and operations while maintaining the knowledge and strategies [11].

\section{A. User interface}

The user interface can effectively help common users get familiar with the strategy establishment procedure, and help users to manage strategies, in the meanwhile, support visiting awareness functionality, proactive precaution functionality and retroactive summary functionality. The functionality of user interface includes:

1) Create and manage strategies. This is the basic functionality of the user interface, where user can complete writing and editing strategies.

2) Manage notices. User could be informed of the execution result of the strategy and receive notice through this interface.
3) Proactive precaution functionality, which helps user understand the effect of the strategies he or she created before they are applied.

4) Retroactive summary functionality, which lets user be aware of which resources have been visited, which strategy leads to the result; and if user is not satisfied with the strategy, the strategy can be revised to avoid of the same situation from happening again.

\section{B. CBR-based learning and conversation technology}

When user creates the strategy for the first time, only a simple and rough default strategy is given by the system. Fig .5 shows that when a conversation case based reasoning (CCBR) functionality is added to the system, before a strategy is given, contextual information can be acquired through conversation process. System could create strategy more suitable with personalized requirements by means of integrating user context with default strategy. Then, the strategy is provided to user to devise. That is referred to as strategy learning [11]. In order to implement this design thought, traditional case-based reasoning (CBR) technology and conversation technology should be integrated to help user refine and revise the strategy step by step. Specific studies include:

CBR. Take strategy as case and study the collection and management of the cases.

1) CBR-based conversation technology. Study how to acquire user's contextual resource via conversations.

2) CBR-based learning technology. Revise and supplement the case library according to the user contextual information.

3) Automatic decision. Study the integration of user strategy and case strategy, importance comparison between user and case strategy decision, and conflict resolution between user and case strategy.

\section{Strategy interpretation system}

Through strategy interpretation system, user can ask about the default strategy and obtain concise answer, by which user could have a better understanding of the strategy. After several revisions of the default strategy, an effective and perfect strategy could be achieved in the end. To implement this interaction mechanism, there are two methods to support the interpretation functionality:

1) In the form of evidence, which based on heuristic method, extracts distinct evidence fragments from the vast history strategies and translates them into a reusable format and formulate a concise answer.

2) In the form of case. Semantic description could better support interpretative sentences which are readable to users. With the case abstract, system could explain the recommended strategy. In addition, system could interpret when user strategy is conflict with strategy recorded in the form of case.

\section{CONCLUSION}

Context-awareness is critical to achieving the vision of pervasive-computing-based smart home, which can adapt to rapidly changing conditions. We propose a conceptual infrastructure employing semantic web technology for 
context representation, reasoning and management. It is expected that this will guide the context-aware smart home design and bridge the divide between present endeavors and aspired smart home with adaptive capabilities. Over time, we envision the context-aware services will significantly improve the quality of our life, benefiting particularly the elderly and disabled people in their everyday life.
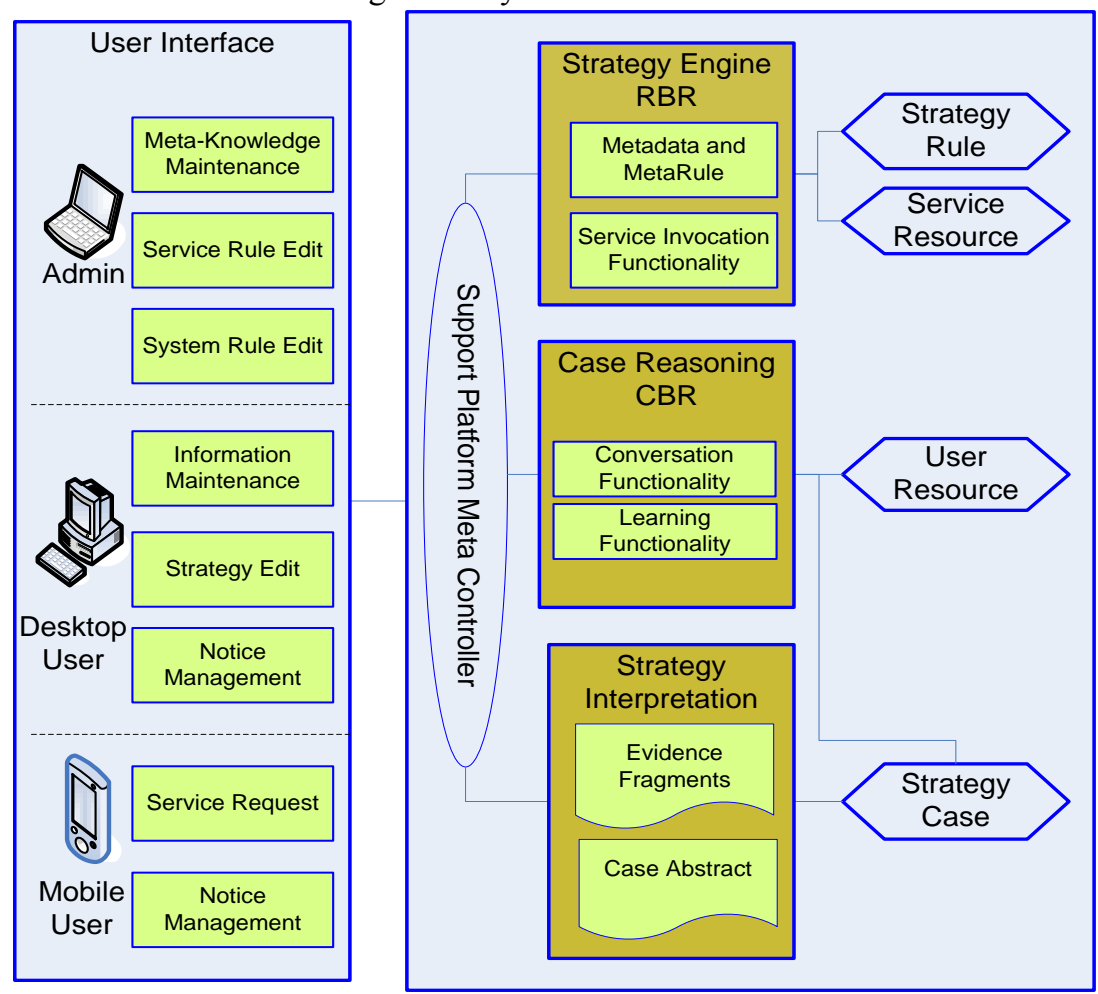

Figure 4. Semantic web knowledge base management platform

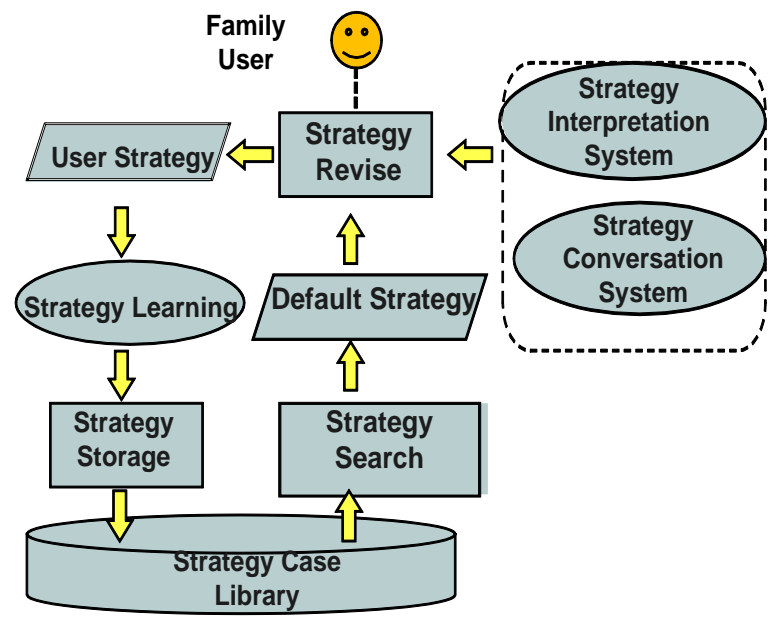

Figure 5. Family user orientated CBR functionality frame

\section{REFERENCES}

[1] Shang-Yuan Chen and Shu-Fen Chang, "A Review of Smart Living Space Development in a Cloud Computing Network Environment," Computer Aided Design \& Applications, 6(4), 2009, 513-527.

[2] T. Yamazaki, "Ubiquitous Home," International Journal of Smart Home,vol.1,no.1,pp.17-22,Dec.2007.
[3] Linzhi Chen, "The study and realization of the intelligent domain name model based on IPv6 home network," Computer Applications and Software, Vol.25, No.9, pp157-159, Sep. 2008. In Chinese.

[4] T.Berners-Lee et al.,"The Semantic Web," Scientific Am., May 2001.

[5] Bricou-Souf N, Newman CR. "Context awareness in health care: a review," International Journal of Medical Informatics 2007;76(1):2-12.

[6] A.K. Dey, Providing Architectural Support for Building, Context-Aware Applications, doctoral dissertation, Georgia Inst. Technology, 2000.

[7] D Wachholder, C Stary, Context-sensitive modeling of input source configuration for evolving intelligent systems, 2014 IEEE Conference on Evolving and Adaptive Intelligent Systems (EAIS), pp. 1-8

[8] A.Kiryakov, B.Popov, I.Terziev, D.Manov, and D.Ognyanoff, "Semantic annotation, indexing, and retrieval," Web Semantics:Science, Services and Agents on the World Wide Web,vol.2,no.1,2011.

[9] Nicklas,D.,Ranganathan,A.and Riboni,D. "A survey of context modelling and reasoning techniques," Pervasive and Mobile Computing,vol.6,pp.161-180. 2010.

[10] LIU Dong, MENG Xiang-Wu, CHEN Jun-Liang, XIA Ya-Mei, "Algorithms for Rule Generation and Matchmaking in Context-Aware System," Journal of Software, Vol.20, No.10, October 2009, pp.2655-2666. In Chinese.

[11] Zhang P. The research and implementation of semantic based Web services discovery [MS. Thesis]. Beijing: Tsinghua University, 2005. In Chinese. 